\title{
Driving the atom by atomic fluorescence: Analytic results for the power and noise spectra
}

\author{
Roberta Marani \\ Institut d'Optique, Boîte Postale 147, 91403 Orsay, France
}

Maurizio Artoni

INFM-European Laboratory of Non-Linear Spectroscopy, Largo Enrico Fermi 2, Florence, Italy and CSIC, Instituto de Estructura de la Materia, C/Serrano 123, 28006 Madrid, Spain

(Received 5 February 1999; published 16 May 2000)

\begin{abstract}
We study how the spectral properties of resonance fluorescence propagate through a two-atom system. Within the weak-driving-field approximation we find that, as we go from one atom to the next, the power spectrum exhibits both subnatural linewidth narrowing and large asymmetries while the noise spectrum of the squeezed quadrature narrows but remains otherwise unchanged. Analytical results for the observed spectral features of the fluorescence are provided and their origin is thoroughly discussed.
\end{abstract}

PACS number(s): 42.50.- p, 32.80.- t

\section{INTRODUCTION}

Since the prediction [1] and observation [2] of the threepeaked fluorescence spectrum of a strongly driven two-level atom, the spectral features of atomic fluorescence have both provided fundamental insight into the photon-atom interaction and revealed a variety of interesting quantum features. Photon antibunching together with sub-Poissonian counting statistics have long been predicted [3-5] and experimentally observed [6-8]. The phenomenon of squeezing in resonance fluorescence has also been the object of a rather intense research activity $[9,10]$. Most of this work has concerned itself with the nonclassical features of fluorescence from an atom excited by a classical light source, typically a coherent light beam. Over the past decade much interest has also been devoted to the issue of an atom driven by a nonclassical light source. In the pioneering work of Gardiner [11], Carmichael [12], Zoller [13], and their co-workers this was studied by considering a model of a two-level atom interacting with a broadband squeezed vacuum. In spite of its simplicity, such a model has brought forward quite a number of interesting predictions, of which the inhibition of the atomic dipole decay due to the reduced quantum fluctuations of the squeezed vacuum reservoir is perhaps the most ubiquitous one. These early results have stimulated much interest [14] on the issue of exciting atomic systems with more realistic forms of nonclassical light such as narrow bandwidth squeezed light [15], antibunched [16] and thermal [17,18] light, and the fluorescence from a high- $Q$ atomic cavity [19]. Among the latest successes in the field, it is certainly worth mentioning a landmark experiment on trapped cesium atoms carried out by Kimble and co-workers [20]; they showed that the twophoton atomic excitation rate, which is typically quadratic with the intensity of the exciting field, may become instead linear for an exciting squeezed light field as long anticipated by the theory $[21,22]$.

Along this line we address the issue of how spectral properties of atomic fluorescence are modified by an atomic medium where succeeding atoms are driven only by the output of the previous one with no feedback. Here, in particular, we study the case of two atoms, one of which is driven by a coherent light beam. We are interested in the power and quadrature noise spectra for both on- and off-resonance excitation and for which we give analytical expressions. The physics of the off-resonant case is much more complex than the resonant one and has not been discussed much in the literature. Our work complements other recent investigations $[16,18,23]$ in which the idea of one atom driven by the fluorescence from another atom is examined. These works consider only on-resonance excitation and look at different features of the output light.

Since nonclassical effects in atomic fluorescence usually take place for weak-field excitations, we calculate the power and quadrature noise (squeezing) spectrum of the fluorescence emitted by each of the two atoms in the weak-field limit. The atomic correlation functions needed to obtain such spectra have recently been derived in [24]; the main expressions for the correlation functions are thus briefly stated in Sec. II while the expressions for the power and noise spectral density of the fluorescent light are explicitly derived in Secs. III and IV. The physical interpretation of these results is also given in these two sections while a summary and conclusions are given in Sec. V.

\section{CORRELATION FUNCTIONS}

Consider a collection of identical two-state atoms, whose energy levels are separated by $\hbar \omega_{0}$, interacting with the radiation field via dipole interaction. Let $\sigma_{-, n}(t)$ be the lowering operator for the $n$th atomic dipole, $R_{n}(t)$ be the population inversion operator and $\mathcal{A}^{(+)}\left(\mathbf{r}_{n}, t\right)$ be the positive frequency part of the vector potential of the electromagnetic field at the position of the $n$th dipole. In terms of the slowly varying dynamical variables $b_{n}(t) \equiv \sigma_{-, n}(t) \exp \left(i \omega_{0} t\right)$ and $A_{n}^{(+)}(t) \equiv \boldsymbol{\mu}_{n} \cdot \mathcal{A}^{(+)}\left(\mathbf{r}_{n}, t\right) \exp \left(i \omega_{0} t\right)$, where $\boldsymbol{\mu}_{n}$ is the induced electric dipole moment, the Heisenberg equations of motion for the atomic operator (in the rotating wave approximation) read as [15]

$$
\begin{gathered}
d b_{n} / d t=-\beta b_{n}+2 \omega_{0} \hbar^{-1} R_{n} A_{n}^{(+)}(t), \\
d R_{n} / d t=-2 \beta\left(R_{n}+1 / 2\right)-\omega_{0} \hbar^{-1}\left[b_{n}^{\dagger} A_{n}^{(+)}(t)+A^{(-)}(t) b_{n}\right] .
\end{gathered}
$$


We denote here by $\beta$ half the natural radiative decay rate. After the $n$th dipole has been excited, the far-field scattered radiation is described by the vector potential (C.G.S. units)

$$
\mathbf{A}^{(+)}(\mathbf{r}, t)=\frac{-i \boldsymbol{\mu}_{n} \omega_{0}}{6 \pi \epsilon_{0} c^{2}\left|\mathbf{r}-\mathbf{r}_{n}\right|} b_{n}\left(t-\left|\mathbf{r}-\mathbf{r}_{n}\right| / c\right)+\mathbf{A}_{\text {free }}^{(+)}(\mathbf{r}, t) .
$$

The first term is the well-known expression for the field radiated by an oscillating dipole located at $\mathbf{r}_{n}$, while the second term is the source-free field contribution that has excited the dipole.

The degree of first- and second-order coherence needed to evaluate the spectrum of the resonance fluorescence and the quadrature noise spectrum can be derived from Eq. (3) using the solutions of the coupled differential equations (1) and (2) for each atomic dipole in the collection. In particular, the system of Eqs. (1) and (2) can be transformed into two coupled integral equations which can be solved, in the weakfield limit, by a perturbative technique when the external driving field only couples to the first atom and each succeeding atom of the collection is driven only by the output of the previous one, with no feedback.

This approach, which is discussed in detail in [24], is applied here to a system of two atoms; the second atom is placed outside the field which drives the first one and the fluorescence feeding back onto the first atom can be clearly assumed to be very small when compared to that driving the second atom. When the field impinging on the first atom is a single-mode coherent state, the steady-state atomic correlation functions, for the first and second atom, are (for positive $\tau)$

$$
\begin{gathered}
\left\langle b_{1}(t)\right\rangle=-\frac{\Omega}{2 \theta_{-}}\left[1-s_{0}\right] \exp \left(i \Delta t+i \theta_{p}\right), \\
\left\langle b_{1}^{\dagger}(t) b_{1}(t+\tau)\right\rangle \\
=\frac{s_{0}}{2}\left[1-2 s_{0}\left(1+\frac{\theta_{+} e^{-\theta_{-} \tau}-\theta_{-} e^{-\theta_{+} \tau}}{4 i \Delta}\right)\right] \exp (i \Delta \tau),
\end{gathered}
$$

$$
\begin{aligned}
\left\langle b_{1}^{\dagger}(t) b_{1}^{\dagger}(t+\tau)\right\rangle= & \frac{s_{0}}{2} \exp \left(-2 i \phi_{+}\right)\left\{1-e^{-\theta_{+} \tau}\right. \\
& \left.-2 s_{0}\left[1-e^{-\theta_{+} \tau}\left(1+\frac{\theta_{+} \tau}{2}\right)\right]\right\} \\
& \times \exp \left[-i \Delta(2 t+\tau)-2 i \theta_{p}\right],
\end{aligned}
$$

and

$$
\begin{aligned}
\left\langle b_{2}(t)\right\rangle= & -\frac{i \Omega \xi_{2}}{2 \theta_{-}}\left[1-s_{0}\left(1+2 s_{1}\right)\right] \exp \left(i \Delta t+i \theta_{p}\right) \\
& \times \exp \left(-i \Delta\left|\mathbf{r}_{2}-\mathbf{r}_{1}\right| / c\right),
\end{aligned}
$$

$$
\begin{aligned}
& \left\langle b_{2}^{\dagger}(t) b_{2}(t+\tau)\right\rangle \\
& =s_{0} s_{1}\left(1-2 s_{0}\left[1+2 s_{1}\left(1+\frac{\theta_{+} e^{-\theta_{-} \tau}-\theta_{-} e^{-\theta_{+} \tau}}{4 i \Delta}\right)\right]\right. \\
& +\frac{s_{0}}{2\left(\beta^{2}+\Delta^{2}\right)}\left\{-\frac{\theta_{-}^{2} e^{-\theta_{+} \tau}}{4 \Delta^{2}}+e^{-\theta_{-} \tau}\right. \\
& \left.\left.\times\left[-\frac{\theta_{+}^{2}\left[\theta_{+}^{2}-7 \theta_{-}^{2}+2\left(\beta^{2}+\Delta^{2}\right)\right]}{16 \beta^{2} \Delta^{2}}-\frac{2 \theta_{+}^{2} \theta_{-} \tau}{4 i \beta \Delta}\right]\right\}\right) \\
& \times \exp (i \Delta \tau) \\
& \left\langle b_{2}^{\dagger}(t) b_{2}^{\dagger}(t+\tau)\right\rangle \\
& =-s_{0} s_{1} \exp \left(-4 i \phi_{+}\right)\left\{\left(1+4 s_{0} s_{1}\right)\right. \\
& \times\left[1-e^{-\theta_{+} \tau}\left(1+\frac{\theta_{+} \tau}{2}\right)\right] \\
& \left.-2 s_{0}\left[1-\frac{e^{-\theta_{+} \tau}}{8}\left(8+5 \theta_{+} \tau+\theta_{+}^{2} \tau^{2}\right)\right]\right\} \\
& \times \exp \left(-i \Delta(2 t+\tau)-2 i \theta_{p}\right) \exp \left(2 i \Delta\left|\mathbf{r}_{2}-\mathbf{r}_{1}\right| / c\right) \text {. }
\end{aligned}
$$

Here $\theta_{ \pm} \equiv \beta \mp i \Delta, \Delta \equiv \omega_{0}-\omega_{L}$ is the detuning between the atomic transition frequency $\omega_{0}$ and the frequency $\omega_{L}$ of the incident laser light, $\phi_{+} \equiv \arg \left(\theta_{+}\right)$, while $\Omega$ and $\theta_{p}$ are the Rabi frequency and phase of the incident field. The parameter $\xi_{2} \equiv\left(\boldsymbol{\mu}_{2} \cdot \boldsymbol{\mu}_{1} \omega_{0}^{2}\right) /\left(6 \pi \epsilon_{0} \hbar c^{2}\left|\mathbf{r}_{2}-\mathbf{r}_{1}\right|\right)$ represents the strength of the interaction between the two dipoles and depends on the direction of propagation of the external pump beam with respect to the interatomic axis and on the interatomic distance $\left|\mathbf{r}_{2}-\mathbf{r}_{1}\right|$. For typical parameters of experimental interest $\xi_{2}$ is much less than $\beta$. We have further introduced the saturation parameters

$$
s_{0} \equiv \frac{\Omega^{2}}{2\left(\beta^{2}+\Delta^{2}\right)} \text { and } s_{1} \equiv \frac{\xi_{2}^{2}}{2\left(\beta^{2}+\Delta^{2}\right)},
$$

which are both much smaller than 1 in the weak-field and far-field limit, respectively.

\section{POWER SPECTRUM}

The incoherent part of the power spectrum of the emitted fluorescence is given by the Fourier transform of the twotime correlation function as

$$
S_{n}^{E}(\mathbf{r}, \omega)=\eta \lim _{t \rightarrow \infty} \int_{-\infty}^{+\infty} d \tau \exp (i \omega \tau)\left\langle\mathcal{E}_{n}^{-}(\mathbf{r}, t), \mathcal{E}_{n}^{+}(\mathbf{r}, t+\tau)\right\rangle,
$$

where $\omega$ represents the scattered frequency, $\eta$ the overall detection efficiency, and $\langle\mathbf{A}, \mathbf{B}\rangle$ denotes the correlation $\langle(\mathbf{A}$ $-\langle\mathbf{A}\rangle) \cdot(\mathbf{B}-\langle\mathbf{B}\rangle)\rangle$. By using the source-field expression for the electric field 


$$
\mathcal{E}_{n}^{-}(\mathbf{r}, t)=\boldsymbol{\epsilon}_{n}(\mathbf{r}) b_{n}^{\dagger}\left(t-\frac{\left|\mathbf{r}-\mathbf{r}_{n}\right|}{c}\right) \exp \left(i \omega_{0} t\right)+\boldsymbol{\mathcal { E }}_{\text {free }}^{-}(\mathbf{r}, t),
$$

where $\boldsymbol{\epsilon}_{n}(\mathbf{r}) \equiv \omega_{0}^{2}\left(\mathbf{r}-\mathbf{r}_{n}\right) \times\left[\boldsymbol{\mu} \times\left(\mathbf{r}-\mathbf{r}_{n}\right)\right] /\left(4 \pi \epsilon_{0} c^{2}\left|\mathbf{r}-\mathbf{r}_{n}\right|^{3}\right)$ is the usual far-field geometrical factor for the dipole radiation, and with the help of results (4)-(9), the spectra of the fluorescence scattered by the first and second atom are, respectively,

$$
\begin{aligned}
S_{1}^{E}(\mathbf{r}, \omega)= & {\left[\eta \frac{\left|\boldsymbol{\epsilon}_{1}(\mathbf{r})\right|^{2}}{\beta}\right] s_{0} } \\
& \times \frac{\Omega^{2} \beta^{2}}{\left\{\beta^{2}+\left[\Delta-\left(\omega-\omega_{L}\right)\right]^{2}\right\}\left\{\beta^{2}+\left[\Delta+\left(\omega-\omega_{L}\right)\right]^{2}\right\}}
\end{aligned}
$$

and

$$
\begin{aligned}
S_{2}^{E}(\mathbf{r}, \omega)= & S_{1}^{E}(\mathbf{r}, \omega)\left|\frac{\boldsymbol{\epsilon}_{2}(\mathbf{r})}{\boldsymbol{\epsilon}_{1}(\mathbf{r})}\right|^{2} \\
& \times\left[4 s_{1}^{2}+2 s_{1} \frac{\left(\beta^{2}+\Delta^{2}\right)}{\left\{\beta^{2}+\left[\Delta-\left(\omega-\omega_{L}\right)\right]^{2}\right\}}\right] .
\end{aligned}
$$

The spectrum (13), which recovers a known result for the fluorescence power spectrum of a weakly driven two-level atom [25,1], consists of two Lorentzian contributions symmetrically displaced with respect to the driving frequency $\omega_{L}$ by an amount $\pm \Delta$. The spectrum (14), proportional to $S_{1}^{E}(\mathbf{r}, \omega)$ through the relative coupling strength of the two atomic dipoles, comprises a constant term and a Lorentzian contribution centered at the value $\Delta$ of the detuning.

It is worth noting here that both the coherent and the incoherent part of the fluorescence scattered off the first atom contribute to the spectrum $S_{2}^{E}(\mathbf{r}, \omega)$. This spectrum depends, in fact, through its definition (11) on the atomic dipole operator $b_{2}$ whose time evolution is governed by the total field $\mathcal{A}^{(+)}$at $\mathbf{r}_{2}$, namely both coherent and incoherent contributions.

The two spectra (13) and (14) are integrated over the whole solid angle, assuming that all emitted light is collected by a detector with perfect efficiency $(\eta=1)$, normalized to the output intensity and plotted in Figs. 1 and 2 for the case of zero and a nonzero detuning $\Delta$.

The finite bandwidth of the two power spectra has a significance here that is different from its significance in the process of atomic spontaneous emission into the vacuum. An excited atom has only a finite amount of stored energy and can radiate only for a finite time, so that the width of the spectral density has to be finite in spontaneous-emission processes. However, in our problem we are dealing with atoms that are continuously excited and the finite size of the linewidth is due to the quantum fluctuations of the field driving the atom, as we will discuss in the following.

Resonant excitation. The role of quantum fluctuations in the fluorescence spectra is already evident in the subnatural linewidth of the light emitted by the first atom for zero de-

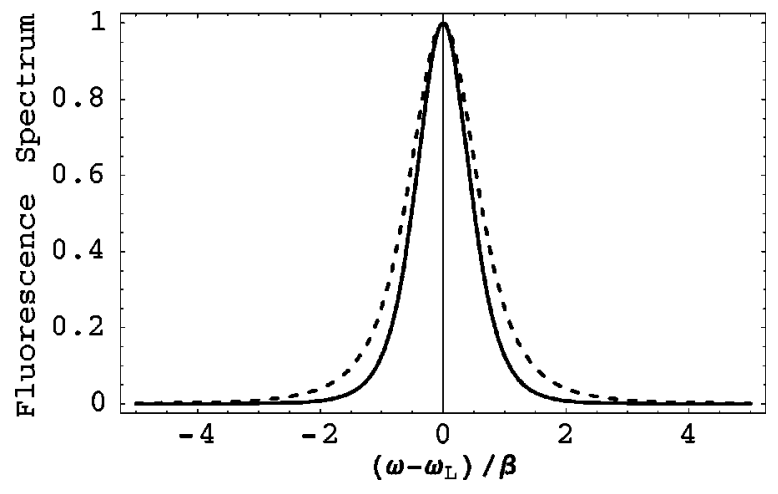

FIG. 1. Spectrum of the fluorescence scattered by the first (dashed line) and by the second (solid line) atom on resonance. For all curves $\Omega / \beta=0.3$. For direct comparison the spectra (12) and (13) have been scaled so as to acquire the value of unit at the atomic transition frequency, i.e., at $\omega=\Delta$.

tuning (dashed curve). This subnatural narrowing is experimentally [26] well established and can be attributed to the atomic dipole fluctuations induced by the coherent exciting field. The spectrum $S_{1}^{E}$ can in fact be decomposed into two Lorentzians describing the fluctuations of the two quadrature phase amplitudes of the induced atomic dipole. Since the fluctuations in one of the quadratures are squeezed, the corresponding Lorentzian gets a negative sign and the two contributions sum up to yield the spectrum (13) with a subnatural linewidth $[27,19,28]$.

The power spectrum of the second atom $S_{2}^{E}$, which goes like the third power of the inverse frequency square, falls off more rapidly than $S_{1}^{E}$ does (Fig. 1). This produces a narrowing in the line shape of the light emitted by the second atom, as compared to that of the first atom, which could originate either from the correlations in the fluorescence incident on the second atom or from the fact that the second atom produces its own squeezing and subsequent narrowing. It can be shown, however, that if the second atom were to be driven by a classical field having the same spectral profile of the fluorescence scattered off the first atom (dashed curve) and made up of monochromatic contributions, thus neglecting the incident field correlations, additional narrowing after the

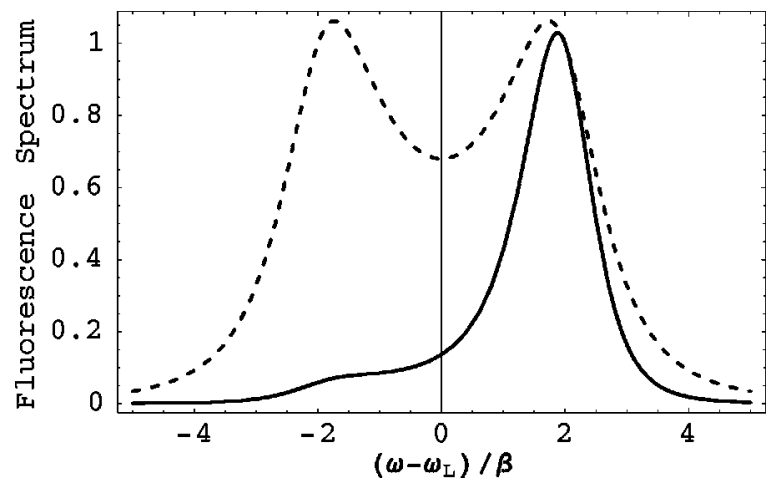

FIG. 2. Spectrum of the fluorescence scattered by the first (dashed line) and by the second (solid line) atom for detuning $\Delta / \beta=2$. The other parameters and the scaling are the same as in Fig. 1 . 
second atom would not occur; the resulting linewidth would be larger [29] than that obtained for monochromatic excitation (dashed curve), suggesting that the power spectrum narrowing after the second atom is mainly due to the presence of quantum correlations (squeezing) in the driving fluorescent light.

Let us also note that the spectral narrowing due to the squeezed incident fluorescence should be phase-dependent; this is, however, not apparent in our case because the squeezing phase in the field exciting the second atom cannot be varied at will but it has a fixed relationship with the phase of the field driving the first atom.

Off-resonant excitation. The case of nonzero detuning exhibits a somewhat more complex physics (Fig. 2). The light emitted by the first atom exhibits a symmetric spectrum with maxima at + and $-\Delta$ from the laser frequency $\omega_{L}$. Such a symmetry originates from the fact that the two Lorentzian contributions in Eq. (13) are equally displaced from $\omega_{L}$ so that for sufficiently large $\Delta$ 's these two components can be far enough apart to make up a well separated doublet whose central dip will decrease as the separation of the two Lorentzians increases. In the limit of vanishing detunings, this well separated doublet merges into a single line centered at resonance.

Physically, the symmetry of the doublet originates from the fact that in the weak-field limit the detuned atom, in order to satisfy energy conservation, responds to the absorption of two laser photons by emitting two photons shifted in frequencies by opposite amounts with respect to $\omega_{L}$. In the dressed-atom approach of resonance fluorescence [30], the symmetric doublet spectrum can be seen to originate from the suppression of the central peak of the fluorescence triplet in the spontaneous transitions between the (first) atom's dressed levels.

The spectrum of the fluorescence emitted by the second atom displays a strong asymmetry in the peak heights in addition to an effect of linewidth narrowing. The dominant term in Eq. (14) not only produces the narrowing but it is also responsible for the asymmetric double-peak structure of the spectrum. Narrowing of the linewidth is again caused by the squeezing in the fluorescence driving the second atom in much the same way as for the undetuned atom.

The fluorescence quenching, which gives rise to the asymmetry, can instead be explained as follows. Because the spectrum emitted by the first atom comprises the incoherent part $S_{1}^{E}$ and the coherent part $S_{1, \operatorname{coh}}^{E}(\omega)=\left[\eta\left|\epsilon_{1}\right| / \beta\right] s_{0}(1$ $\left.-2 s_{0}\right) \beta \delta\left(\omega-\omega_{L}\right)$, the light impinging on the second atom draws, according to Eq. (13), contributions from a range of frequencies scattered over the interval $\left(\omega_{0}-2 \Delta-\beta, \omega_{0}\right.$ $+\beta$ ) with a peak at $\omega_{L}$ (coherent scattering) and two side peaks centered at about $\omega_{0}-2 \Delta$ and $\omega_{0}$ (incoherent scattering). The coherently scattered radiation from the first atom is monochromatic and yields a symmetric contribution much like that of the first atom driven by the monochromatic laser beam; this is the very small term $\left(s_{1} \ll 1\right)$ on the right-hand side of Eq. (14). The incoherently scattered radiation from the first atom, on the other hand, gives rise to the asymmetric and larger term in Eq. (14), which mainly enhances the resonant peak; this contribution arises instead from the single- photon absorption-emission processes. In fact, each incident $\omega$ scatters into a two-peak spectrum having one peak on resonance and the other at $2 \omega-\omega_{0}$, so that for each $\omega$ the resonant component of the spectrum is enhanced resulting in a stronger on-resonance peak while the off-resonance components are smeared over the spectrum yielding the observed quenching.

\section{NOISE SPECTRUM}

The resonance fluorescence radiated by a two-level atom driven by coherent light exhibits the phenomenon of squeezing [31]. This effect is typically revealed by using a phasesensitive homodyned detection scheme [24,32]. In such a scheme the atomic fluorescence is mixed by means of a symmetric beam splitter with the highly coherent light of a local oscillator of the same frequency. Squeezing in the signal beam can be assessed by measuring the normally ordered and time-ordered intensity correlation function

$$
\lambda\left(t_{1}, t_{2}\right) \equiv \frac{\left\langle\mathcal{T}: I\left(t_{1}\right) I\left(t_{2}\right):\right\rangle}{\left\langle I\left(t_{1}\right)\right\rangle\left\langle I\left(t_{2}\right)\right\rangle}-1,
$$

where $I$ is the intensity of the mixed field at the detector.

When the local oscillator is made much stronger than the fluorescent signal, the intensity correlation function for the fluorescence scattered from the $n$th atom is given by

$$
\begin{aligned}
\lambda_{n}\left(\mathbf{r}, t, t+\tau, \psi_{n}\right)= & 2 \frac{\left|\boldsymbol{\epsilon}_{n}(\mathbf{r})\right|^{2}}{\left|\boldsymbol{\epsilon}_{\mathrm{LO}}(\mathbf{r})\right|^{2}} \operatorname{Re}\left\{\exp \left[i\left(\omega_{\mathrm{LO}}-\omega_{0}\right) \tau\right]\right. \\
& \times\left\langle b_{n}^{\dagger}\left(t_{n, \mathbf{r}}\right), b_{n}\left(t_{n, \mathbf{r}}+\tau\right)\right\rangle+\exp \left(2 i \psi_{n}\right) \\
& \times \exp \left[-i\left(\omega_{\mathrm{LO}}-\omega_{0}\right)(2 t+\tau)\right] \\
& \left.\times\left\langle b_{n}^{\dagger}\left(t_{n, \mathbf{r}}\right), b_{n}^{\dagger}\left(t_{n, \mathbf{r}}+\tau\right)\right\rangle\right\}
\end{aligned}
$$

where $t_{n, \mathbf{r}} \equiv t-\omega_{0}\left|\mathbf{r}-\mathbf{r}_{n}\right| / c$ is the typical retardation time, $\psi_{n} \equiv \phi_{\mathrm{LO}}+(\pi / 2)-\left(\omega_{0}\left|\mathbf{r}-\mathbf{r}_{n}\right| / c\right)$, and $\phi_{\mathrm{LO}}$ is the local oscillator phase. In homodyne detection the local oscillator frequency $\omega_{\mathrm{LO}}$ is equal to the laser frequency $\omega_{L}$. In the stationary regime $\lambda_{n}(\mathbf{r}, t, t+\tau)$ depends only on $\tau$, and its Fourier transform yields the quadrature noise spectrum

$$
S_{n}^{N}\left(\mathbf{r}, \omega, \psi_{n}\right)=\eta\langle I\rangle \int_{-\infty}^{+\infty} d \tau \exp (i \omega \tau) \lambda_{n}\left(\mathbf{r}, \tau, \psi_{n}\right) .
$$

The phase of the local oscillator $\phi_{\mathrm{LO}}$ is chosen so as to yield maximum squeezing for fixed values of the Rabi frequency and detuning. This occurs when the intensity correlation function (16) acquires the highest negative value for $\tau=0$, so that $\psi_{1}=\phi_{+}$and $\psi_{2}=2 \phi_{+}+(\pi / 2)$ for the first and second atom, respectively, as can be inferred from Eqs. (6) and (9). In general, the contribution from the first term on the righthand side of Eq. (16) to the noise spectrum (17) can be written as $\left[S_{n}^{E}\left(\mathbf{r}, \omega+\omega_{L}\right)+S_{n}^{E}\left(\mathbf{r},-\omega+\omega_{L}\right)\right] / 2$, while we will denote by $\widetilde{S}_{n}\left(\mathbf{r}, \omega, \psi_{n}\right)$ the contribution coming from the last term on the right-hand side of Eq. (16). Since for the first atom the power spectrum is symmetric [see Eq. (13)], we obtain 


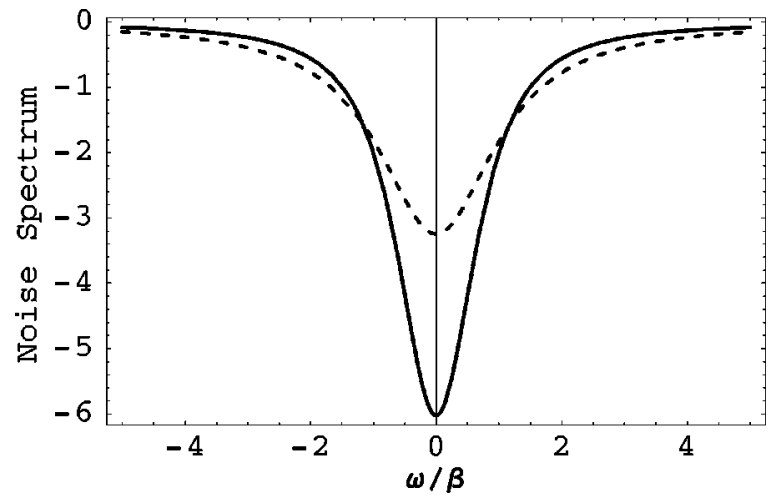

FIG. 3. Normalized noise spectrum (squeezed quadrature) of the fluorescence scattered by the first atom (dashed line) and by the second atom (solid line) for no detuning, $\Omega / \beta=0.3, \psi_{1}=\phi_{+}, \psi_{2}$ $=2 \phi_{+}+(\pi / 2)$, and $\xi_{2} / \beta=0.02$.

$$
S_{1}^{N}(\mathbf{r}, \omega, \pm)=S_{1}^{E}\left(\mathbf{r}, \omega+\omega_{L}\right) \pm \widetilde{S}_{1}\left(\mathbf{r}, \omega, \psi_{1}\right)
$$

where the plus (minus) sign in the third argument of the noise spectrum corresponds to the squeezed (antisqueezed) quadrature, and

$$
\begin{aligned}
\widetilde{S}_{1}\left(\mathbf{r}, \omega, \psi_{1}\right)= & {\left[\frac{\eta\left|\boldsymbol{\epsilon}_{1}(\mathbf{r})\right|^{2}}{\beta}\right]\left(2 s _ { 0 } ^ { 2 } \left[\frac{\beta^{2}+\Delta^{2}-\omega \Delta}{\left[\beta^{2}+(\Delta-\omega)^{2}\right]^{2}}\right.\right.} \\
& \left.\left.+\frac{1}{\beta^{2}+(\Delta-\omega)^{2}}\right]-2 s_{0} \frac{1}{\beta^{2}+(\Delta-\omega)^{2}}\right) \\
& +\{\omega \rightarrow-\omega\} .
\end{aligned}
$$

For the second atom we obtain instead

$$
\begin{aligned}
S_{2}^{N}(\mathbf{r}, \omega, \pm)= & \frac{S_{2}^{E}\left(\mathbf{r}, \omega+\omega_{L}\right)+S_{2}^{E}\left(\mathbf{r},-\omega+\omega_{L}\right)}{2} \\
& \pm \widetilde{S}_{2}\left(\mathbf{r}, \omega, \psi_{2}\right),
\end{aligned}
$$

where

$$
\begin{aligned}
\tilde{S}_{2}\left(\mathbf{r}, \omega, \psi_{2}\right)= & \left.\frac{\left.\eta\left|\boldsymbol{\epsilon}_{2}(\mathbf{r})\right|^{2}\right]}{\beta}\right]-4 s_{1}^{2} s_{0}^{2}\left[\frac{2\left(\beta^{2}+\Delta^{2}-\omega \Delta\right)}{\left[\beta^{2}+(\Delta-\omega)^{2}\right]^{2}}\right. \\
& \left.+\frac{1}{\beta^{2}+(\Delta-\omega)^{2}}\right]+4 s_{1} s_{0}^{2}\left[\frac{\beta^{2}+\Delta^{2}-2 \omega \Delta}{\left[\beta^{2}+(\Delta-\omega)^{2}\right]^{3}}\right. \\
& \left.+\frac{2 \beta^{2}+6 \Delta^{2}-3 \omega \Delta}{\left[\beta^{2}+(\Delta-\omega)^{2}\right]^{3}}+\frac{3}{2\left[\beta^{2}+(\Delta-\omega)^{2}\right]}\right] \\
& \left.-s_{1} s_{0}\left[\frac{2\left(\beta^{2}+\Delta^{2}-\omega \Delta\right)}{\left[\beta^{2}+(\Delta-\omega)^{2}\right]^{2}}+\frac{1}{\beta^{2}+(\Delta-\omega)^{2}}\right]\right) \\
& +\{\omega \rightarrow-\omega\} .
\end{aligned}
$$

The spectrum (18) is consistent with the weak-field limit of a known expression for the noise spectrum [10,32], while Eq. (20) is a new result. In Fig. 3 we plot the spectrum (18)

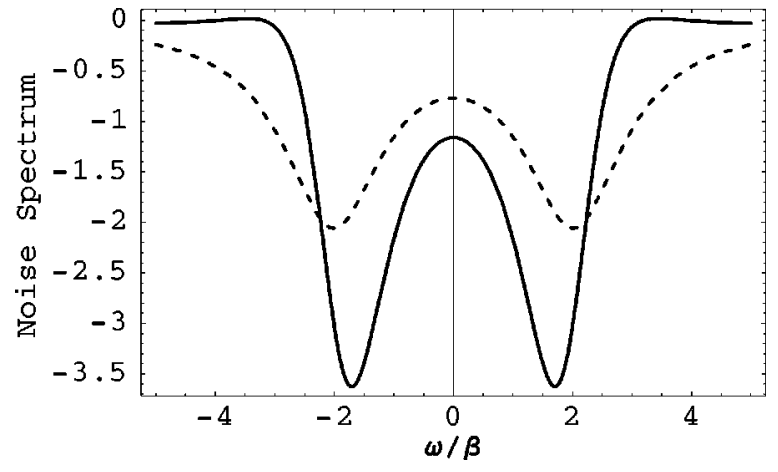

FIG. 4. Normalized noise spectrum (squeezed quadrature) of the fluorescence scattered by the first atom (dashed line) and by the second atom (solid line) for $\Delta / \beta=2, \Omega / \beta=0.3, \xi_{2} / \beta=0.02, \psi_{1}$ $=\phi_{+}$, and $\psi_{2}=2 \phi_{+}+(\pi / 2)$.

and (20) (squeezed quadrature) for an undetuned atom and suitably normalized to the outgoing intensity while the same is done for a detuned atom in Fig. 4. The outgoing intensity is given by $\left\langle b_{n}^{\dagger}(t) b_{n}(t)\right\rangle\left|\epsilon_{n}(\mathbf{r})\right|^{2}$. We first observe that for both detuned and undetuned atoms the noise bandwidth narrows as the fluorescence propagates from one atom to the next because in general the atom-radiation coupling lengthens the characteristic correlation time of the scattered fluorescence [24]. This is also consistent with the findings of Gardiner et al. [33] for a two-level atom excited by squeezed light from a degenerate parametric amplifier.

Furthermore, one can see that for an undetuned atom maximum squeezing is seen to arise in a narrow band around $\omega=0$, where it reaches an absolute value of about $12 \%$ after the first atom, confirming the result found in $[9,10,32]$, but reduces to $5 \times 10^{-3} \%$ after the second atom. These percent values are obtained multiplying Eqs. (18) and (20) by the appropriate outgoing intensity. The very small noise reductions after the second atom are due to the fact that the effective Rabi frequency for the second atom is about $10^{-2}$ times smaller than that for the first atom. For a detuned atom, on the other hand, the amount of squeezing is generally smaller with the largest values occurring over sidebands centered around $\pm \Delta$. For $\Delta=2 \beta$ a maximum absolute squeezing of about $1 \%$ is observed after the first atom but drops again to $1 \times 10^{-4} \%$ after the second one (Fig. 4).

Finally we see that the fluorescence from the first atom exhibits homogeneous squeezing (i.e., squeezing is present at all frequencies), while for detunings greater than $2 \beta$, the fluorescence from the second atom is squeezed only for frequencies between the side minima. This is illustrated in Fig. 5 , which shows a contour plot of the noise spectrum $S_{2}^{N}(\mathbf{r}, \omega,+)$ normalized to the outgoing intensity as a function of frequency and detuning. The white area represents positive values (indicating that the light is not squeezed at the corresponding frequency) while darker colors indicate increasing negative values.

\section{CONCLUSIONS}

In this paper we calculate the effect of propagation through an atomic medium on the spectral properties of reso- 


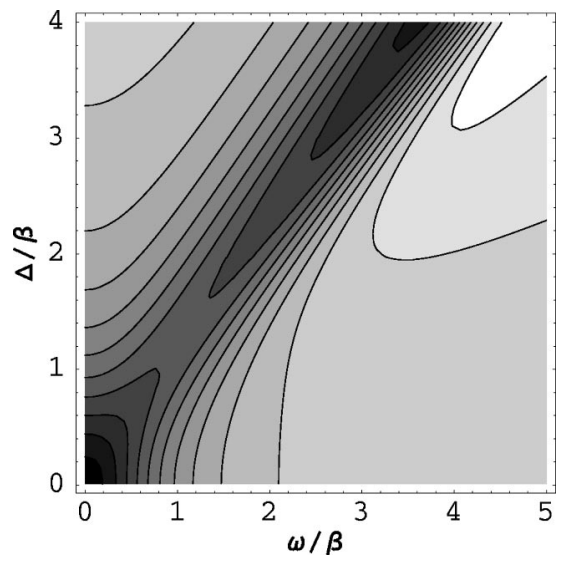

FIG. 5. Contour plot of the noise spectrum (squeezed quadrature) of the second atom for $\Omega / \beta=0.3$ and $\xi_{2} / \beta=0.02$ as a function of frequency and detuning. We take $\psi_{2}=2 \phi_{+}+(\pi / 2)$. The contour lines represent values of the spectrum from -5 to 0.5 , in 0.5 increments. Lighter areas correspond to higher values. The spectra are normalized to the outgoing intensity.

nance fluorescence. For a two-atom system, where the first atom is excited either by a resonant or nonresonant coherent light source and the second one only by the fluorescence scattered by the first one (no feedback), we provide analytical expressions for the fluorescence power spectrum and for the fluorescence quadrature noise spectrum (squeezing). Such a simple system offers the advantage of analytical results which enable one to stress the basic differences between the spectral signatures of the fluorescence scattered by an atom driven by a classical (first) and fluorescent (second) light field. In the weak-field regime we observe narrowing of the linewidth both in the power and noise spectra and an appreciable fluorescence quenching effect after the second atom.

The mechanisms for line narrowing after the first and after the second atom are different. The subnatural linewidth narrowing in the power spectra after the first atom is described by Eq. (13) and originates from the fact that the interaction between the atom and a coherent laser source produces squeezing of the atomic dipole fluctuations in phase with the mean induced dipole moment. Further narrowing after the second atom, which is described by the square bracket term on the right-hand side of Eq. (14), results from the presence of squeezing in the fluorescent radiation that excites it.

The quadrature noise spectrum of the first and second atom fluorescence is instead described by the expressions (18) and (20), and reductions of the noise bandwidth from one atom to the next are due to the lengthening of correlation time as the fluorescence propagates from one atom to the other.

Unlike the effect of linewidth narrowing, which occurs for both detuned and undetuned atoms, quenching of the fluorescence takes place only for nonvanishing detunings after the second atom. Such a quenching results from the asymmetry introduced by the second term in the large square brackets in Eq. (14) and it is here interpreted as due to the enhancement of the resonant component of the finitebandwidth radiation impinging on the second atom.

It is worth mentioning finally that for appropriate values of the detuning the two-atom system that we examine here is remarkably similar to that of an atom driven by a bichromatic field with one resonant and one off-resonant component of the same intensity. The dynamical and spectral features of such an apparently simple atomic configuration have been investigated in a surprisingly small number of experiments; yet such a system is expected to exhibit quite a rich physics that ranges from parametric [34] and Rabi subharmonic resonances [35] to two-photon optical gain and lasing [36]. The compact analytical results derived here may then be of help in understanding the not so obvious spectral features of the fluorescence from a two-level atom under bichromatic and weak excitation [37], and work in this direction is underway [38].

\section{ACKNOWLEDGMENTS}

The authors are grateful to C. Cabrillo, P. Zhou, and S. Swain for enlightening discussions.
[1] B. R. Mollow, Phys. Rev. 188, 1969 (1969).

[2] H. M. Shuda and C. R. Stroud, J. Phys. B 7, L198 (1974).

[3] H. J. Carmichael and D. Walls, J. Phys. B 9, 1199 (1976).

[4] H. J. Kimble and L. Mandel, Phys. Rev. A 13, 2123 (1976).

[5] L. Mandel, Opt. Lett. 4, 205 (1979).

[6] H. J. Kimble, M. Dagenais, and L. Mandel, Phys. Rev. Lett. 39, 691 (1977).

[7] J. Cresser et al., in Dissipative Systems in Quantum Optics, edited by R. Bonifacio (Springer-Verlag, Berlin, 1982), Vol. 27.

[8] R. Short and L. Mandel, Phys. Rev. Lett. 51, 384 (1983).

[9] R. Loudon, Opt. Commun. 49, 24 (1984).

[10] M. J. Collett, D. F. Walls, and P. Zoller, Opt. Commun. 52, 145 (1984).

[11] C. W. Gardiner, Phys. Rev. Lett. 56, 1917 (1986).
[12] H. J. Carmichael, A. S. Lane, and D. F. Walls, Phys. Rev. Lett. 58, 2539 (1987).

[13] H. Ritsch and P. Zoller, Phys. Rev. A 38, 4657 (1988).

[14] Recent reviews on this subject can be found, for example, in [18] and in H. Hegerfeldt, Quantum Semiclassic. Opt. 9, 961 (1997).

[15] R. Vyas and S. Singh, Phys. Rev. A 45, 8095 (1992).

[16] C. W. Gardiner, Phys. Rev. Lett. 70, 2269 (1993).

[17] G. P. Hildred, S. S. Hassan, R. R. Puri, and R. K. Bullough, J. Phys. B 16, 1703 (1983).

[18] C. W. Gardiner and A. S. Parkins, Phys. Rev. A 50, 1792 (1994).

[19] P. R. Rice and H. J. Carmichael, J. Opt. Soc. Am. B 5, 1661 (1988).

[20] N. P. Georgiades et al., Phys. Rev. Lett. 75, 3426 (1995); Z. 
Ficek and P. Drummond, Phys. Today 50 (9), 34 (1997).

[21] Gea-Banacloche, Phys. Rev. Lett. 62, 1603 (1989).

[22] J. Javanainen and P. L. Gould, Phys. Rev. A 41, 5088 (1990).

[23] P. Kochan and H. J. Carmichael, Phys. Rev. A 50, 1700 (1994).

[24] M. Artoni and R. Marani, Phys. Rev. A 54, 2280 (1996).

[25] H. J. Kimble and L. Mandel, Phys. Rev. A 15, 689 (1977).

[26] F. Y. Wu, R. E. Grove, and S. Ezekiel, Phys. Rev. Lett. 35, 1426 (1975).

[27] Already in Ref. [1] Mollow notes that the form of the spectrum (12) (zero detuning) can be attributed to the addition of two Lorentzian components, one of which appears with negative weight.

[28] S. Swain and P. Zhou, Opt. Commun. 123, 310 (1996).
[29] This is also expected from general results of linewidth broadening due to nonmonochromatic sources. See, e.g., Ref. [1].

[30] C. Cohen-Tannoudji and S. Reynaud, J. Phys. B 10, 145 (1977).

[31] D. F. Walls and P. Zoller, Phys. Rev. Lett. 47, 709 (1981).

[32] Z. Y. Ou, C. K. Hong, and L. Mandel, J. Opt. Soc. Am. B 4, 1574 (1987).

[33] C. W. Gardiner, A. S. Parkins, and M. J. Collett, J. Opt. Soc. Am. B 4, 1683 (1987).

[34] P. Thomann, J. Phys. B 13, 1111 (1980).

[35] S. Papademetriou et al., Phys. Rev. A 53, 997 (1996).

[36] D. J. Gauthier et al., Phys. Rev. Lett. 68, 464 (1992).

[37] C. C. Yu et al., Phys. Rev. A 56, R4381 (1997).

[38] R. Marani and M. Artoni (unpublished). 